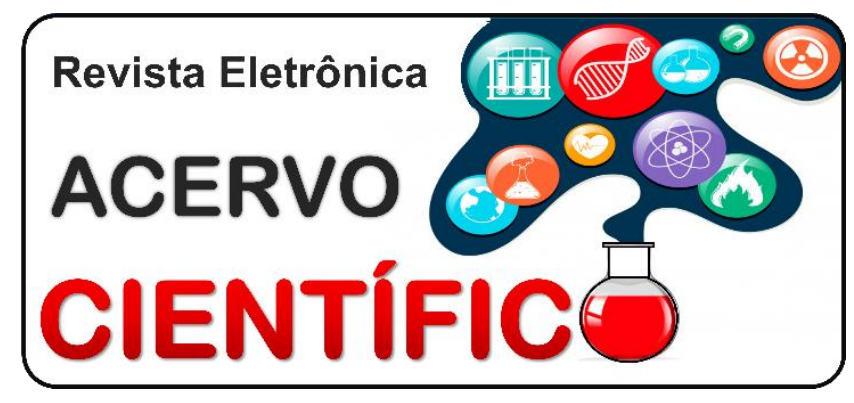

\section{REVISÃO BIBLIOGRÁFICA}

Recebido em: 10/2020

Aceito em: 11/2020

Publicado em: 12/2020

\title{
Impactos do tratamento hormonal e não hormonal sobre a sintomatologia vasomotora de mulheres climatéricas
}

\author{
The impacts of hormonal and non-hormonal treatments on vasomotor symptoms of \\ climacteric women
}

\section{Los impactos de los tratamientos hormonales y no hormonales en los síntomas vasomotores de las mujeres climatéricas}

Isabella Ferraz Ferreira ${ }^{1 *}$, Danielle Fiorin Ferrari Novais ${ }^{1}$, Ana Clara Mendonça de Carvalho ${ }^{3}$, Emily Thomazini Mombrine ${ }^{4}$, Fernanda Mazarin Agostinho ${ }^{5}$, Isabella Pereira Sabino ${ }^{6}$, Laiana Alves Andrade $^{7}$, Rafaela Inácio Nassif ${ }^{5}$, Rayssa Ferreira Lopes $^{7}$, Ricardo de Araújo Mello Júnior ${ }^{8}$.

\begin{abstract}
Resumo: $\mathrm{O}$ artigo busca analisar os diferentes tratamentos hormonais e alternativos, que visam atenuar as ocorrências vasomotoras e sua relação com a melhora na qualidade de vida das mulheres climatéricas. $O$ climatério é definido como o período de transição entre a fase reprodutiva e não reprodutiva da mulher, marcado pela diminuição da secreção de estrogênio. Os desequilíbrios hormonais que ocorrem durante esse período, são responsáveis pelas manifestações que prejudicam a qualidade de vida da mulher, como os sintomas vasomotores, dentre eles fogachos e sudorese, sendo estes os fenômenos mais comuns no diagnóstico do climatério. Nesse período de transição, cerca de $25 \%$ das mulheres irão necessitar de terapia para o alívio dos sintomas. O tratamento durante essa fase deverá ser individualizado para cada caso específico, avaliando sempre as características apresentadas por cada paciente, e pode variar entre Terapia de Reposição Hormonal (TRH), não hormonal e tratamento não farmacológico. A TRH utilizada para o controle dos fenômenos climatéricos possui variação de resultados, conforme o esquema de utilização das drogas, e não é aconselhado o uso prolongado, devido ao aparecimento de reações adversas, como a trombose venosa.
\end{abstract}

Palavras-chave: Climatério, Fogachos, Terapia de reposição hormonal.

Abstract: The article seeks to analyze the different hormonal and alternative treatments that aim to mitigate vasomotor events and their relationship with the improvement in the quality of life of climacteric women. The

\footnotetext{
${ }^{1}$ Universidade de Vassouras (UV), Vassouras - RJ. *Email: ferrazisabella@hotmail.com

2 Faculdade Multivix, Vitória - ES.

${ }^{3}$ Universidade de Fortaleza (UNIFOR), Fortaleza - CE.

4 Universidade Vila Velha (UVV), Vila Velha - ES.

5 Universidade de Ribeirão Preto (UNAERP), Ribeirão Preto - SP.

${ }^{6}$ Centro Universitário Claretiano (CEUCLAR), Rio Claro - SP.

7 Centro Universitário Atenas (UniAtenas), Paracatu - MG.

8 Universidade de Uberaba (UNIUBE), Uberaba - MG.
} 
climacteric is defined as the transition period between the woman's reproductive and non-reproductive phase, marked by the decrease in estrogen secretion. The hormonal imbalances that occur during this period are responsible for the manifestations that impair the woman's quality of life, such as vasomotor symptoms, among them hot flushes and sweating, these being the most common phenomena in the diagnosis of climacteric. During this transition period, about $25 \%$ of women will need therapy for symptom relief. Treatment during this phase should be individualized for each specific case, always evaluating the characteristics presented by each patient, and can vary between Hormone Replacement Therapy (HRT), non-hormonal and non-pharmacological treatment. The HRT used to control climacteric phenomena has variation in results according to the drug use scheme and prolonged use is not advised, due to the appearance of adverse reactions, such as venous thrombosis.

Keywords: Climacteric, Hot flashes, Hormone replacement therapy.

Resumen: El artículo busca analizar los diferentes tratamientos hormonales y alternativos que tienen como objetivo mitigar los eventos vasomotores y su relación con la mejora en la calidad de vida de las mujeres climatéricas. El climaterio se define como el período de transición entre la fase reproductiva y no reproductiva de la mujer, marcado por la disminución de la secreción de estrógenos. Los desequilibrios hormonales que se producen durante este período son responsables de las manifestaciones que perjudican la calidad de vida de la mujer, como los síntomas vasomotores, entre ellos los sofocos y la sudoración, siendo estos los fenómenos más comunes en el diagnóstico de climatérico. Durante este período de transición, alrededor del $25 \%$ de las mujeres necesitarán terapia para aliviar los síntomas. El tratamiento durante esta fase debe individualizarse para cada caso específico, evaluando siempre las características que presenta cada paciente, y puede variar entre terapia hormonal sustitutiva (THS), tratamiento no hormonal y no farmacológico. La THS utilizada para controlar los fenómenos climatéricos varía según el esquema de uso del fármaco y no se recomienda su uso prolongado, debido a la aparición de reacciones adversas, como la trombosis venosa.

Palabras clave: Climaterio, Sofocos, Terapia de reemplazo de hormonas.

\section{INTRODUÇÃO}

O ciclo hormonal é divido em três fases, sendo elas: menarca, climatério e menopausa. A menarca é utilizada como indicador de maturação biológica, e marca o início da vida reprodutiva da mulher (SANTOS MLB, et al., 2015). Ao fim da secreção hormonal dos ovários, há interrupção fisiológica dos ciclos menstruais, que representa o início da menopausa e o fim da fase reprodutiva feminina (FERREIRA VN, et al., 2013).

O climatério constitui o período de transição entre esses dois estágios e, geralmente, ocorre entre os 40 a 65 anos de idade, tendo como marco a diminuição da secreção de estrogênio (AKIYOSHI M, et al., 2011). Este intervalo se subdivide em pré-menopausa, perimenopausa e pós menopausa, sendo a perimenopausa o período do acontecimento dos sinais e sintomas que podem também definir a fase climatérica (MELO G, COSTA A, 2018).

Durante esse momento, há irregularidades do ciclo menstrual, devido ao início da perda da função ovariana, além de distúrbios variados, como insônia, alterações psicológicas e na libido, que prejudicam a qualidade de vida das mulheres (ABDI F, et al., 2016; SAYAN S, et al., 2018). A perda da libido está relacionada a uma redução de testosterona e a lentidão da lubrificação vaginal é referente a queda da produção de estrogênio (VALENÇA CN, et al., 2010).

Os sintomas vasomotores (SVM), compreendidos por fogachos, tonturas e sudorese, são incômodas e se apresentam de forma recorrente no climatério. As ondas de calor são os sintomas que mais contribuem para o diagnóstico deste período, e ocorrem devido à maior expressão dos receptores de serotonina, os quais estão associados à disfunção do centro termorregulador do hipotálamo (JIMÉNEZ AP, et al.,2018). 
O tratamento usual é pautado na terapia hormonal (TRH), contudo, há mulheres que optam pelo tratamento alternativo. A TRH mais utilizada é uma combinação entre estrogênio e progestágenos, que se ligam a receptores intracelulares e atuam sobre a transcrição gênica, promovendo redução dos sintomas em $80 \%$. Estudos apontam que esse tratamento pode aumentar o risco de doenças cardiovasculares, osteoporose e câncer de mama, fatores estes que contribuem para o maior interesse por terapias alternativas, entre elas: uso de folhas de amoreira-preta, acupuntura e homeopatia (DREWE J, et al., 2015).

O objetivo do presente estudo consiste em revisar os tratamentos hormonais e alternativos que reduzem os sintomas vasomotores, buscando a relação destes com a melhora da qualidade de vida das mulheres climatéricas.

\section{REVISÃO BIBLIOGRÁFICA}

\section{Definição e epidemiologia}

Segundo a Organização Mundial de Saúde (OMS), o climatério representa a transição fisiológica entre a fase reprodutiva (menacme) e não reprodutiva da mulher, a qual acomete a faixa etária dos 40 aos 65 anos. Nesse período, acontece a menopausa, que é estabelecida como a interrupção permanente da menstruação, caracterizada após 12 meses consecutivos de amenorreia (ausência de menstruação) (CURTA J e WEISSHEIMER AM, 2020).

$\mathrm{Na}$ menacme, o ciclo menstrual da mulher é controlado pelo eixo hipotálamo-hipófise-ovário. O hipotálamo induz a produção do hormônio liberador das gonadotrofinas $(\mathrm{GnRH})$ pela pré-hipófise, que estimula a síntese de hormônio luteinizante (LH) e hormônio folículo estimulante (FSH), responsáveis por regular a função ovariana, através do estímulo à secreção de estrogênio e progesterona pelo ovário. Dessa forma, o período menstrual regular é desencadeado por altas concentrações de $\mathrm{FSH}$ e quantidades moderadas de LH. Num primeiro momento, o estrógeno age no eixo hipotálamo-hipófise para inibir a expressão do $\mathrm{GnRH}$, através de feedback negativo, e, consequentemente, bloquear a liberação de FSH e LH. Posteriormente, sinaliza para a pré-hipófise a necessidade de sintetizar e disponibilizar, de forma abrupta, os hormônios gonadotrópicos, especialmente o LH, resultando em seu pico no plasma (COELHO S, et al., 2015).

A ovulação é um fenômeno que ocorre aproximadamente no 14ำ dia e propicia o desenvolvimento do corpo lúteo, que passa a secretar altas quantidades de estrogênio e progesterona. Esses hormônios tornam a inibir a pré-hipófise, o que ocasiona uma profunda depleção de FSH e LH. Sem o impulso desses dois hormônios, o corpo lúteo involui e, por resultado, diminui as concentrações de progesterona e estrogênio. Nessa circunstância, ocorre a menstruação provocada pela privação dos hormônios ovarianos (COELHO S, et al., 2015).

Em contrapartida, na menopausa ocorre a redução progressiva da função ovariana, que desencadeia o declínio dos hormônios ovarianos, estrogênio e progesterona. Através do feedback sob o eixo hipotálamohipófise, há aumento das gonadotrofinas hipofisárias, FSH e LH, acarretando cessação da menstruação. Sendo assim, é possível afirmar que a alta concentração de FSH é a primeira indicação laboratorial do climatério inicial (ISLAM MR, et al., 2015). Dessa forma, é possível dividir o climatério em três fases: prémenopausa, que se inicia especialmente após os 40 anos, com redução da fertilidade em mulheres com o ciclo menstrual regular; perimenopausa, que origina-se dois anos antes do último ciclo menstrual e dura até um ano após; e pós-menopausa, que é estabelecida um ano após o último ciclo menstrual (CURTA J e WEISSHEIMER AM, 2020).

Normalmente, a irregularidade menstrual é o primeiro sintoma dessa transição fisiológica, sendo a causa de maior relevância a amenorreia hipotalâmica funcional associada à redução da secreção do $\mathrm{GnRH} e$ desregulação do eixo hipotálamo-hipófise-adrenal. A ocorrência desses distúrbios hormonais pode levar ao desenvolvimento de infertilidade, doenças cardíacas e diabetes mellitus do tipo 2 (BAE J, et al., 2018). Além das modificações hormonais nas mulheres, esse período também experimenta alterações menstruais e morfológicas, como atrofia mamária e urogenital, ressecamento da pele e da mucosa vaginal. 
Há também o predomínio de sintomas sistêmicos, como nervosismo, irritabilidade, insônia, depressão, sudorese, palpitações, alterações na libido, dor muscular e esquelética, dispareunia e aumento da reabsorção óssea (MANDUJANO MR, et al., 2019; JIMENEZ AT e RINCON JT, 2018; BEDELL S, et al.,2013). Também se observa também a influência de aspectos psicossociais, sexuais, familiares, afetivos e ocupacionais da mulher que irão predispor ao aumento dos sintomas climatéricos (AYERS B, et al., 2010).

Com o aumento contínuo da expectativa de vida, há um número crescente de mulheres expostas aos sintomas climatéricos, especialmente aos SVM, representadas por fogachos e sudorese profusa noturna. Por meio de uma revisão sistemática, é possível estimar que estas manifestações incidem em 14\% a 51\% das mulheres antes da transição menopausal, em 35\% a $50 \%$ na transição e $30 \%$ a $80 \%$ após a menopausa (GRAVENA A, et al., 2013).

As alterações hormonais anteriormente descritas, que ocorrem na fase climatérica, causam instabilidade no centro termorregulador hipotalâmico, promovendo ativação dos mecanismos que propiciam perda de calor corporal, a fim de diminuir a temperatura central. Desse modo, há aumento da temperatura periférica da pele, desencadeando a ocorrência dos fogachos (BARCIA J, 2011).

Os fogachos, principais representantes dos SVM, consistem em sensação súbita e transitória de calor moderado ou intenso, que se espalha pelo tórax, pescoço e face. Os fatores de risco que contribuem para a ocorrência destes, incluem: idade da menopausa, baixa escolaridade, trabalho mal remunerado, tabagismo e alto índice de massa corporal (IMC). Essas ondas de calor normalmente ocorrem diariamente, entre 4 a 10 anos após a menopausa, com um pico por volta de um ano (AYERS B e HUNTER MS, 2012).

Durante a peri e pós-menopausa, há também predominância da sudorese profusa (aglomerado vasomotor), que ocorre preferencialmente no período noturno, deixando a paciente em estado de vigília. Um estudo na Escócia, com mulheres entre 45 a 54 anos, demonstrou alguns fatores de risco aos suores noturnos, que englobam: tabagismo, possível depressão, dificuldade para dormir, ondas de calor, relato de um ou dois sintomas sexuais e utilização de estratégias de modificação do estilo de vida para controlar os sintomas (DUFFY OK, et al., 2012).

Esse trabalho mostrou que, diferente dos fogachos, as pacientes que apresentavam o IMC alto, no valor de $30 \mathrm{~kg} / \mathrm{m}^{2}$, eram menos favoráveis a relatar suores noturnos do que aquelas que tinham o IMC no valor de referência $\left(25 \mathrm{~kg} / \mathrm{m}^{2}\right)$. O alto peso corporal representa fator de proteção para a sudorese profusa noturna, devido à quantidade de estrogênio produzido pelo tecido adiposo periférico (DUFFY OK, et al., 2012).

Diante do exposto, estima-se que, aproximadamente, $25 \%$ das mulheres necessitarão de terapia para alívio da sintomatologia durante o período de climatério, devido às consequências negativas sob a qualidade de vida. Deve-se, contudo, individualizar o tratamento de acordo com cada paciente, levando em consideração as indicações e restrições inerentes à cada caso (AYERS B e HUNTER MS, 2012; HICKEY et al., 2017).

\section{Tratamento}

No que tange à terapêutica do climatério, esta deverá ser individualizada de acordo com a sintomatologia apresentada por cada paciente. No caso dos SVM, os fogachos representam a maior parte das queixas, e podem ser beneficiados por terapia de reposição hormonal (TRH), não hormonal e de tratamento não farmacológico (JIMÉNEZ AP e RINCÓN JM, 2018).

A base da TRH pode ser feita com estrogênio, estradiol, hidroxiprogesterona, levonorgestrel, entre outros, mas sem eficácia comprovada a longo prazo por alguns estudos. Como uma forma de terapia alternativa para o tratamento desses sintomas, consta a Tibolona, um esteróide sintético que apresenta resultados satisfatórios no alívio de SVM, além de melhorar a atrofia urogenital e precaver a diminuição da massa óssea (JIMÉNEZ AP e RINCÓN JM, 2018; PARDINI D, 2014).

Em relação ao tratamento não hormonal, tem-se os Inibidores de Recaptação de Serotonina (ISRS), que se mostraram bastante eficazes no alívio de sintomas de calor, além de atuarem sobre as queixas 
associadas à instabilidade emocional, depressão, ansiedade, angústia, visto que aumentam a quantidade de serotonina disponível. Alternativamente aos ISRS, pode-se citar a classe dos anticonvulsivantes, como os análogos do GABA: Gabapentina e Pregabalina, fármacos que têm sido categóricos em relação aos sintomas de fogachos nas pacientes. Apesar de apresentarem efeitos colaterais, como vertigem, cefaleia, tremedeiras, sono, enjôo, dentre outros, a adesão foi tolerável e, com isso, os resultados se mostraram válidos nas mulheres que não se adaptam e/ou são restritas ao uso dos hormonais (JIMÉNEZ AP e RINCÓN JM, 2018; SHAN D, et al., 2020).

Os tratamentos não farmacológicos abrangem mudanças de estilo de vida, que incluem evitar o tabagismo, aumento de ingesta hídrica e proteica, além da prática de atividade física. Nesta, deve-se priorizar os exercícios aeróbicos, com uma frequência de 30 minutos por dia e de, pelo menos, 3 vezes na semana. A obtenção de uma alimentação mais equilibrada e de hábitos de vida mais saudáveis, melhoram diversos aspectos e, consequentemente, a qualidade de vida (JIMÉNEZ AP e RINCÓN JT, 2018; SHAN D, et al., 2020).

De acordo com alguns estudos sobre a medicina tradicional chinesa, a acupuntura tem se apresentado como um benefício na amenização de fogachos, melhorando a regularidade e o autocontrole das ondas de calor. Ademais, ressalta-se melhora nas desordens do sono em mulheres pós menopausa, além de contribuir para o bem estar da paciente, entretanto, ainda não é designada para substituir a terapia hormonal. A prática de loga se mostrou eficiente na diminuição das ondas de calor e na transpiração, pois trabalha o corpo, a mente, a postura e a atenção. Não se domina ainda o mecanismo de como o loga interfere nas práticas de cada doença, mas há indícios que ele é eficaz no sistema nervoso e endócrino (EISENHARDT S e FLECKENSTEIN J, 2016; BARRA A, et al., 2014).

De acordo com Padilha $\mathrm{M}$, et al. (2010) há trabalhos relacionando as folhas de amoreira preta como insumo farmacêutico, podendo ser utilizada e indicada como uma alternativa de terapia hormonal, tendo eficácia nas queixas de fogachos. Além disso, possui um mecanismo de ação que reduz os efeitos de oxidação e inflamação e, ainda, atua na diminuição da concentração de glicose no sangue (hipoglicemiantes) e da aparição de bactérias e fungos (microbianos) no organismo.

O uso da homeopatia vem sendo cada vez mais estudado, como mostra um questionário realizado no México com 31 mulheres com a idade variando entre 45 a 60 anos, abrangendo os sintomas característicos do climatério, como a presença de fogachos, ressecamento vaginal, mudanças repentinas de humor, palpitações, entre outros. Os medicamentos mais eficazes foram Lachesis trigonocephalus e Pulsatilla nigricans, os quais minimizam os devidos sintomas e se sobressaíram benéficos no tratamento (MANDUJANO M, et al., 2019).

\section{Impactos da THR frente aos sintomas climatéricos}

A TRH é prescrita para as mulheres que estão passando da fase reprodutiva para a menopausa, a fim de melhorar e prevenir os sintomas citados acima. Essa terapia tem variação nos seus resultados de acordo com o esquema de utilização das drogas, e pode ser feita com estrogênio, progestágenos ou com uso de outras drogas, como a tibolona. Esta última age diminuindo os níveis de globulina ligadora de hormônios sexuais (SHBG), promovendo assim, um aumento na testosterona livre (POLISSENI AF, et al.,2012; JIMENEZ AP e RINCON JT, 2018).

Com este aumento hormonal, há efeitos benéficos nas funções sexuais, como desejo e lubrificação, favorecendo assim uma melhora na qualidade de vida das mulheres climatéricas. Tanto o estrogênio, quanto o progestogênio, se ligam aos receptores intracelulares, com efeito na transcrição ou repressão gênica. Tal fato controla a regulação hormonal do centro termorregulador no hipotálamo, e, consequentemente, promove redução dos SVM (POLISSENI AF, et al.,2012; JIMENEZ AP e RINCON JT,2018).

Os SVM ocorrem principalmente devido à redução de estrógeno e de serotonina circulantes, e correspondem a um conjunto de manifestações clínicas, como sensação de calor intenso, sudorese (principalmente noturna), e, até instabilidade emocional e distúrbios de sono. Tais sintomas podem acabar 
afetando a qualidade de vida das mulheres nesse período de transição. Levando em conta que a principal explicação fisiopatológica para a ocorrência dessas manifestações é a interrupção do fornecimento de estrogênio, a TRH é a indicação terapêutica primordial para o alívio desses sintomas (ABDI F, et al., 2016)

A administração de estrogênios na TRH, também é eficaz na prevenção do aumento da reabsorção óssea associada à menopausa (ABDI F, et al., 2016). Com a reposição hormonal, ainda há prevenção de alterações geniturinárias, visto que age inibindo a diminuição da produção de colágeno e secreções, impedindo assim, a fragilidade da mucosa vaginal, a má lubrificação e a atrofia urogenital (JIMENEZ AT e RINCON JT, 2018).

Apesar de auxiliar nos sintomas climatéricos, a TRH não deve ser utilizada por tempo prolongado, devido ao surgimento de reações adversas como trombose venosa, ainda que o mecanismo pelo qual isso ocorre não esteja bem estabelecido. É aconselhado que o uso desses hormônios ocorra nos primeiros 10 anos de menopausa, em mulheres que não apresentem contraindicações e que tenham idade inferior a 60 anos. Além disso, recomenda-se iniciar a TRH com baixas doses, para reduzir a probabilidade de efeitos colaterais. Sendo assim, é de fundamental importância que TRH seja individualizada para cada paciente, levando em conta os riscos-benefícios e as contraindicações (JIMENEZ AP e RINCON JT,2018).

\section{Complicações do climatério e da TRH}

É reconhecido que o período da menopausa pode ser assintomático, mas, na maioria das vezes, os sintomas estão presentes de forma e intensidade variáveis em cada mulher, sendo os cardiovasculares, em especial, a hipertensão arterial, o motivo pelo qual foi analisado se essas pacientes poderiam se beneficiar de TRH. Além disso, pacientes jovens, com IMC aumentado, período de menopausa diminuído, circunferência abdominal elevada e aumento de colesterol LDL e triglicerídeos, apresentam maior chance de ocorrência de fogachos. Sendo assim, deve-se ressaltar a importância de incrementar atividade física e a modificação do estilo de vida (DUFFY OK, et al, 2012; SAYAN S, et al., 2018).

As mulheres climatéricas apresentam, muitas vezes, desânimo, tristeza e outros distúrbios psicológicos, em decorrência das alterações hormonais e sistêmicas. Em concordância, o uso de TRH tem sido uma alternativa eficaz para melhorar a qualidade de vida das pacientes.

Além disso, mostrou-se uma discreta redução do peso naquelas em uso de estrogênio associado a progesterona em relação àquelas que não fazem uso. Segundo estudos, a via transdérmica é mais segura, pois não altera lipidograma, coagulograma e perfil pressórico (GRAVENA A, et al., 2013; MOUNIERVEHIER, et al., 2019).

Em contrapartida, alguns estudos evidenciam que o uso de terapia estrogênica isolada está associado a um discreto aumento de peso, além de aumentar os riscos de câncer endometrial e hiperplasia endometrial, ao contrário da terapia combinada, que oferece proteção ao endométrio (GRAVENA A, et al., 2013; PARDINI D, 2013).

Outro possível malefício da TRH é o aumento da ocorrência de eventos tromboembólicos. Esse risco tende a ser maior no primeiro ano de uso da terapia e em pacientes obesas, com mais de 60 anos de idade, portadoras de trombofilias, história de cirurgia prévia e imobilizações. Nessas pacientes, é indicado que a terapia de reposição estrogênica seja feita por via transdérmica. Se a opção se der por terapia combinada, o mais indicado é progesterona micronizada ou didrogesterona (PARDINI D, 2013).

Ainda não há evidências que relacionam o tabagismo com maior ocorrência de SVM, no entanto, o cigarro possui efeitos contrários ao estrogênio, por meio da elevação das transaminases que são encarregadas pelo processo de metabolismo. Outrem, podem ocorrer infecções (ação inflamatória), interferindo também em efeitos relacionados a trombrogênese, principalmente na formação da trombose $\mathrm{e}$ placas de gordura na artéria, promovendo um elevado fator de risco para doenças cardiovasculares (SAYAN S, et al., 2018; ROSA E SILVA ACJS, MELO AS, 2010).

De acordo com alguns trabalhos, após uma análise do perfil nutricional das mulheres no climatério, envolvendo fontes de proteína, cálcio, lipídios, carboidratos, vitamina $D$, provou-se uma carência de cálcio. 
De certo modo, contribui ainda mais para a ocorrência de distúrbio ósseo na mulher, promovendo a osteopenia e até mesmo o avanço da osteoporose (LIMA L, et al., 2016).

\section{CONSIDERAÇÕES FINAIS}

O climatério é um período fisiológico marcado por múltiplas mudanças no organismo feminino, e a manifestação de sinais e sintomas sistêmicos, como fogachos, atrofia mamária e genital, alterações na libido e mudanças psicossociais, refletem as alterações hormonais. O tratamento deve ser individualizado, de acordo com a sintomatologia de cada paciente, podendo ser farmacológico, através da TRH ou de tratamento não hormonal, além das alternativas não farmacológicas, as quais abrangem mudanças no estilo de vida, ambas visando a melhora da qualidade de vida da mulher climatérica. Ainda, é importante ressaltar que, devido às modificações hormonais e aos tratamentos, o organismo feminino torna-se suscetível à complicações, que incluem aumento do colesterol, além de maior risco ao desenvolvimento de síndrome metabólica, câncer, hiperplasia endometrial e eventos tromboembólicos, sendo de fundamental importância o acompanhamento contínuo dessas mulheres por profissionais de saúde.

\section{REFERÊNCIAS}

1. ABDI F, et al. Hormone Therapy for Relieving Postmenopausal Vasomotor Symptoms: A Systematic Review. Arch Iran Med., 2016; 19(2): 141-146.

2. AKIYOSHI M, et al. Relationship between estrogen, vasomotor symptoms, and heart rate variability in climacteric women. J Med Dent Sci, 2011; 58: 49-59.

3. AYERS B, et al. The impact of attitudes towards the menopause on women's symptom experience: a systematic review. Maturitas, 2010; 65(1): 28-36.

4. AYERS B, HUNTER MS. Health-related quality life of women with menopausal hot flushes and night sweats. Climacteric, 2012; 16(2): 235-239.

5. BARCIA J. Síntomas vasomotores en la mujer climatérica. Rev Cubana Obstet Ginecol, 2011; 37(4): 577-592.

6. BARRA A, et al. Terapias alternativas no climatério. Revista Feminina, 2014; 42: 27-31.

7. BEDELL S, et al. The pros and cons of plant estrogens for menopause. The Journal of Steroid Biochemistry and Molecular Biology, 2014; 139: 225-236.

8. CURTA JC. Perceptions and feelings about physical changes in climacteric women. Revista Gaúcha de Enfermagem, 2019; 41: e20190198.

9. DREWE J, et al. A systematic review of non-hormonal treatments of vasomotor symptoms in climacteric and câncer patients. Springer Plus, 2015; 65(4): 1-29.

10. DUFFY OK, et al. Factors associated with reporting classic menopausal symptoms differ. Climacteric, 2012; 16(2): 240-251.

11. DUFFY OK, et al. The impact and management of symptoms experienced at midlife: a community-based study of women in northeast scotland. Bjog: An International Journal of Obstetrics \& Gynaecology, 2012; 119(5): 554-564.

12. EISENHARDT S, FLECKENSTEIN J. Traditional Chinese medicine valuably augments therapeutic options in the treatment of climacteric syndrome. Arch Gynecol Obstet, 2016; 294: 193-200.

13. FERREIRA VN, et al. Menopausa: Marco biopsicossocial do envelhecimento feminino. Psicologia \& Sociedade, 2013; 25(2): 410-419.

14. GRAVENA A, et al. Sintomas climatéricos e estado nutricional de mulheres na pós-menopausa usuárias e não usuárias de terapia hormonal. Revista Brasileira de Ginecologia e Obstetrícia, 2013; 35(4): 178-184.

15. ISLAM MR, et al. Prevalence of menopausal symptoms in Asian midlife women: a systematic review. Climacteric, 2014; 18(2): 157-176.

16. JIMENEZ AP, RINCON JT. Climaterio y Menopausia. Revista de la Facultad de Medicina de la UNAM, 2018; 61(2): 51-58.

17. LIMA L, et al. Perfil do consumo alimentar e da relação cálcio/proteína de mulheres no climatério. Nutrición Clínica Y Dietética Hospitalaria, 2016; 36(2): 55-62.

18. MANDUJANO M, et al. Evaluación del Tratamiento Homeopático de Mujeres en Climaterio entre los 45 y 60 años con la Menopause Rating Scale (MRS). La Homeopatia de México, 2019; 88(716): 28-35.

19. MELO G, COSTA A. Influence of climacteric symptoms in women's quality of life: integrative review. Hum Reprod Arch., 2018; 32(3): 1-9. 
20. MOUNIER-VEHIER C. HTA et traitement hormonal de la menopause. La Presse Médicale, 2019; 48: $1295-1300$.

21. PADILHA M, et al. Estudo farmacobotânico das folhas de amoreira-preta, Morus nigra L., Moraceae. Revista Brasileira de Farmacognosia, 2010; 4(20): 621-626.

22. PARDINI D. Terapia de reposição hormonal na menopausa. Arq Bras Endocrinol Metab. 2014; 58(2): $172-181$.

23. POLISSENI AF, et al. Efeitos de um regime combinado contínuo de terapia hormonal de baixa dosagem (estradiol e acetato de noretindrona) e tibolona na qualidade de vida em mulheres pós-menopáusicas sintomáticas: um estudo duplo-cego, randomizado. Tese (Doutorado em Saúde) - Área de concentração em Saúde Brasileira. Universidade Federal de Juiz de Fora, Juiz de Fora, 2013; 98 p.

24. ROBERTS H, HICKEY M. Managing the menopause: an update. Maturitas, 2016; 86: 53-58.

25. ROSA E SILVA ACJS, MELO AS. A importância da via de administração na terapia hormonal do climatério. Revista Feminina. 2010; 38(6).

26. SANTOS MLB, et al. Insatisfação corporal e qualidade de vida durante a menarca e sua relação com a renda familiar e o índice de massa corporal: Um estudo longitudinal. Motricidade, 2015; 11(12): 75 - 84.

27. SAYAN S, et al. Relationship between vasomotor symptoms and metabolic syndrome in postmenopausal women. Journal of International Medical Research, 2018; 46(10): 4157-4166.

28. SHAN D, et al. Efficacy and safety of gabapentin and pregabalin in patients with vasomotor symptoms: a systematic review and meta-analysis. American Journal of Obstetrics \& Gynecology, 2020; 222: 564-579.

29. SOUZA N, et al. Relação entre terapia de reposição hormonal no climatério e o desenvolvimento de neoplasias. Brazilian Journal of Surgery and Clinical Research, 2018; 25: 135-143.

30. VALENÇA CN, et al. Mulher no Climatério: reflexões sobre desejo sexual, beleza e feminilidade. Saúde Soc. 2010; 19(2): 273-285. 